\title{
Subthreshold Activation of the Superior Colliculus Drives Saccade Motor Learning
}

\author{
Robijanto Soetedjo, Albert F. Fuchs, and Yoshiko Kojima \\ Department of Physiology and Biophysics and Washington Regional Primate Research Center, University of Washington, Seattle, Washington 98195-7330
}

How the brain learns and maintains accurate precision movements is currently unknown. At times throughout life, rapid gaze shifts (saccades) become inaccurate, but the brain makes gradual adjustments so they again stop on target. Previously, we showed that complex spikes (CSs) in Purkinje cells of the oculomotor cerebellum report the direction and amplitude by which saccades are in error. Anatomical studies indicate that this error signal could originate in the superior colliculus (SC). Here, we deliver subthreshold electrical stimulation of the SC after the saccade lands to signal an apparent error. The size of saccades in the same direction as the simulated error gradually increase; those in the opposite direction decrease. The electrically adapted saccades endure after stimulation is discontinued, exhibit an adaptation field, can undergo changes in direction, and depend on error timing. These electrically induced adaptations were virtually identical with those produced by the visually induced adaptations that we report here for comparable visual errors in the same monkeys. Therefore, our experiments reveal that an additional role for the SC in the generation of saccades is to provide a vector error signal that drives dysmetric saccades to adapt. Moreover, the characteristics of the electrically induced adaptation reflect those of error-related CS activity in the oculomotor cerebellum, suggesting that CS activity serves as the learning signal. We speculate that CS activity may serve as the error signal that drives other kinds of motor learning as well.

\section{Introduction}

The cerebellum has been implicated in the adaptation of movements to reduce actual or perceived errors in performance. For example, if normal subjects wear prism glasses that displace the visual scene, their finger pointing initially is inaccurate, but, over just a few trials, they reduce their motor dysmetria and point directly at a target. If, however, patients with cerebellar lesions wear displacement prisms, they never regain their pointing accuracy (Martin et al., 1996).

Like finger pointing, saccades rapidly point the direction of gaze at interesting targets. Saccades also undergo adaptation after extraocular muscle injury (Optican and Robinson, 1980) or when an apparent error is induced by surreptitiously displacing the target as the eye "saccades" toward it (McLaughlin, 1967; Straube et al., 1997). If saccades consistently appear to either fall short or overshoot the target, their amplitudes gradually increase or decrease, respectively. Lobules VIc and VII of the cerebellar vermis are essential for such saccade adaptation because humans (Straube et al., 2001) or monkeys (Takagi et al., 1998; Barash et al., 1999) fail to adapt dysmetric saccades when these oculomotor areas are lesioned.

What neuronal mechanism could be involved in such saccade motor learning? One model implicates vermis Purkinje cells

Received Aug. 31, 2009; revised 0ct. 1, 2009; accepted 0ct. 22, 2009.

This work was supported by National Institutes of Health (NIH) Grants EY00745 (A.F.F.) and EY019258 (R.S.) and Grant RR00166 from the National Center for Research Resources (NCRR), a component of the NIH. Its contents are solely the responsibility of the authors and do not necessarily represent the official views of NCRR or NIH. We received valuable comments from Steve Bierer, Elena Buzunov, Leo Ling, Adrienne Mueller, Farrel Robinson, and Avery Weiss on the first version of this manuscript.

Correspondence should be addressed to Dr. Robijanto Soetedjo, Washington National Primate Research Center, Box 357330, University of Washington, Seattle, WA 98195-7330. E-mail: robi@u.washington.edu.

DOI:10.1523/JNEUROSCI.4296-09.2009

Copyright $\odot 2009$ Society for Neuroscience ～0270-6474/09/2915213-10\$15.00/0
( $\mathrm{P}$ cells), which discharge frequent simple spikes (SSs) and occasional complex spikes (CSs). In this scenario, a persistent error in saccade performance alters the frequency of CSs, which, in turn, promotes changes in saccade-related SS activity (Marr, 1969; Albus, 1971; Ito, 2000). These changes influence oculomotor nuclei in the brainstem to alter saccade size gradually and reduce the error. Two lines of evidence support such a model. First, signals producing CSs in the vermis are relayed through the inferior olive, and lesions of this pathway cause enduring saccade dysmetrias that never are corrected (Waespe and Baumgartner, 1992; Helmchen et al., 1994). Second, the probability of CS occurrence in P cells of lobules VIc and VII signals both the direction and size of the error of dysmetric saccades (Soetedjo and Fuchs, 2006; Soetedjo et al., 2008). But does this error signal actually drive saccade adaptation?

We address this question by stimulating the CS pathway electrically to create an artificial error signal. CSs in the oculomotor vermis arise from activity in the contralateral medial accessory olive (Kralj-Hans et al., 2007), which, in turn, receives a dense projection from the contralateral superior colliculus (SC) (Huerta and Harting, 1984). Because the SC is topographically organized according to the position of a target on the retina and the size and direction of targeting saccades (Goldberg and Wurtz, 1972; Robinson, 1972; Sparks and Mays, 1980), stimulation at different SC loci simulates errors with different vectors. As a saccade is underway, we turn off the target and replace the usual visual error that occurs after a dysmetric saccade with electrical stimulation of the SC, at the time when the natural CS error signal would occur. Such stimulation produces changes in saccade amplitude with all of the features of normal behavioral adaptation. 


\section{Materials and Methods}

General animal preparation and recording. Two rhesus monkeys (5.8-6.0 $\mathrm{kg}$ ) were used in this study. Under sterile surgical procedures, we implanted a scleral search coil on one eye to measure eye position with an electromagnetic technique (Fuchs and Robinson, 1966; Judge et al., 1980) and stabilization lugs on the skull to prevent head movements. After recovery, they were trained to track a target spot that jumped every $1-1.5 \mathrm{~s}$ in a dimly lit room. They were required to fixate the target for $0.4-1 \mathrm{~s}$, not including their reaction time, to obtain a drop of applesauce reward. The target was a dot image $\left(0.4^{\circ}\right.$ diameter $)$ of a red laser reflected from a pair of $X-Y$ mirror galvanometers onto either a cylindrical or a tangent screen, 44 or $68 \mathrm{~cm}$, respectively, away from the monkey's eyes. The target always appeared within $\pm 20^{\circ}$ of straight ahead.

After they were trained to track the jumping target spot accurately for $>2 \mathrm{~h}$, we implanted a SC recording chamber, which was positioned on the midline, angled $38^{\circ}$ posterior from the coronal plane, and aimed at 15 $\mathrm{mm}$ dorsal and $1 \mathrm{~mm}$ posterior to the center of the interaural line. After recovery, we recorded eye and target position, as well as the extracellular activity of SC neurons with homemade tungsten microelectrodes, which we coated with iron particles to reduce their impedance to $100-200 \mathrm{k} \Omega$ at $1 \mathrm{kHz}$. Unit activity was bandpass filtered between $300 \mathrm{~Hz}$ and $10 \mathrm{kHz}$ and digitized at 50,000 samples/s. This unit channel also was used to record the electrical stimulation pulse train. The eye and target position recordings were low-pass filtered at $300 \mathrm{~Hz}$ and digitized at 1000 samples/s.

We identified the superficial layer of the SC by neurons that discharged a burst $\sim 60-70 \mathrm{~ms}$ after the appearance of a target in their visual receptive field, but did not discharge for the subsequent targeting saccade (Goldberg and Wurtz, 1972). Approximately 1-1.5 mm deeper (1.2 \pm $0.4 \mathrm{~mm}$ ), we encountered neurons with both a visual and subsequent saccade-related response, which we attributed to the visuomotor cells in the intermediate layer. Once we had identified this intermediate layer, we stimulated $(50 \mu \mathrm{A} ; 600 \mathrm{~Hz} ; 100 \mu$ s negative monophasic pulse; $50 \mathrm{~ms}$ train duration) to determine the direction and amplitude of saccades characteristic of that SC site. Within this range, we usually found visuomotor or quasivisual neurons (Mays and Sparks, 1980). Before the stimulation, the monkey maintained fixation on a target straight ahead for 2-5 s. The target then was extinguished, and $100 \mathrm{~ms}$ later the stimulus train was delivered. The target remained off for $500 \mathrm{~ms}$. We collected 8-10 evoked saccades and measured the average direction and amplitude of their vector. We considered this vector as the "error vector" signaled by the SC. In different experiments, we stimulated at different sites in the SC topographic map (Robinson, 1972) so that the error vectors represented a variety of directions and amplitudes.

Conditions common to all experiments. First, we estimated the vector of the putative error signal at the stimulus site as the saccade elicited by suprathreshold stimulation. Then, the parameters of the stimulus train (either frequency, current, or both) were gradually reduced until stimulation evoked saccades on $<10 \%$ of trials. Note that, if the electrically induced saccade adaptation had resulted solely from those $<10 \%$ evoked saccades, the amount of amplitude decrease and increase would have been $<15 \pm 4$ and $<13 \pm 3 \%$, respectively, of the full amplitude change obtained after the entire course of the stimulation. Therefore, we conclude that the $<10 \%$ evoked saccades could not have contributed significantly to the adaptation. We maintained this subthreshold condition throughout the experiment by continually monitoring eye position online. In 31 experiments, the stimulus train lasted $100 \mathrm{~ms}$. For that stimulus duration, subthreshold parameters ranged from 10 to $30 \mu \mathrm{A}$ (average $\pm \mathrm{SD}, 18 \pm 5 \mu \mathrm{A}$ ) and $100-225 \mathrm{~Hz}$ (average, $165 \pm 32 \mathrm{~Hz}$ ). In nine experiments in which the train lasted only $5-10 \mathrm{~ms}$, the subthreshold range was $40-100 \mu \mathrm{A}$ (average, $60.6 \pm 18.8 \mu \mathrm{A}$ ) and $425-800 \mathrm{~Hz}$ (average, $572 \pm 104 \mathrm{~Hz}$ ). We delivered the stimulus train after every targeting saccade.

In different experiments, the stimulus train followed delays ranging from 0 to $600 \mathrm{~ms}$ from the end of a saccade (Fig. $1 \mathrm{~A}$ ). The direction and size of the targeting saccade relative to the error vector and the duration of the delay varied from experiment to experiment as described below. However, for every experiment, a targeting saccade turned off the target
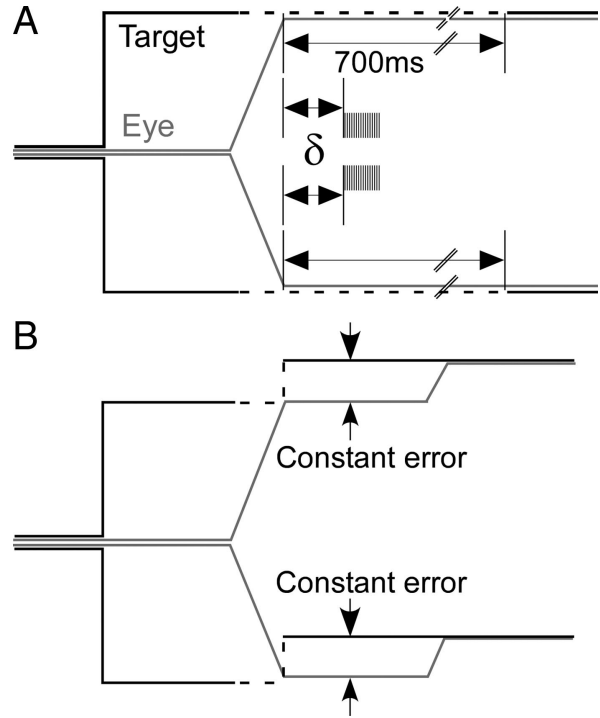

Figure 1. Methods to induce electrically and visually induced adaptation. $\boldsymbol{A}$, For electrically induced adaptation, occurrence of a targeting saccade extinguished the target for $700 \mathrm{~ms}$ (dashed line) and a subthreshold stimulus train (thin black lines) was delivered $\delta \mathrm{ms}$ after the end of each saccade. The position of the extinguished target was clamped to the position of the eye (gray traces) at the end of each saccade. $\boldsymbol{B}$, For visually induced adaptation, occurrence of a targeting saccade extinguished the target until the end of the saccade (dashed line). Then the target was illuminated at a fixed offset (constant error) from the eye position (gray traces) at the end of the saccade, either forward to produce an amplitude increase (top traces) or backward to produce an amplitude decrease (bottom traces).

for $700 \mathrm{~ms}$ (dashed target traces) to eliminate any postsaccadic visual error signal. To minimize the visual error when the target was again turned on, we placed the extinguished target on the eye position at the end of the saccade so when it was reilluminated the error was near zero.

After each stimulation experiment, we performed behavioral adaptation experiments with the same error conditions (Fig. $1 B$ ). These behavioral data usually were taken $2 \mathrm{~d}$ after the stimulation experiment to ensure that any previous adaptation had dissipated. The monkey made saccades in the same way as in the previous stimulation experiment, but now the target was displaced relative to the landing eye position of the saccade. To do so, we turned the target off during each saccade (dashed target traces). While the target was off, we first stabilized the extinguished target on the eye position and then jumped it relative to the eye. The jump was adjusted to create the same visual error vector that stimulation of the site in the SC had simulated in a previous stimulation experiment (Fig. $1 B$ ). Therefore, adaptation produced by stimulation and behavior could be directly compared.

We now will consider the experimental details that are specific to the three different experimental conditions we examined.

Stimulation to induce an amplitude change. The first goal of our study was to induce amplitude decrease and increase adaptation by delivering SC subthreshold stimulation. After we had determined the vector of the putative error signal at an SC site by suprathreshold stimulation, we required the monkey to track 10 or $15^{\circ}$ target steps in the same and opposite directions of the error vector. We delivered a $100 \mathrm{~ms}$ train that started $80 \mathrm{~ms}$ after the saccade landed to mimic the timing distribution of CSs (Soetedjo et al., 2008). We expected that stimuli delivered after saccades in the same direction as the error signal would cause saccade amplitude to increase, whereas stimuli delivered after saccades in the opposite direction would cause it to decrease. Stimulation was delivered after every saccade for at least 200 trials in each direction. Behavioral adaptation data were collected by applying the same visual error vector relative to eye position at the end of each saccade.

Before the stimulation, we collected saccades to target steps of $5,10,15$, and $20^{\circ}$ in both adapted directions, and to $15^{\circ}$ target steps at \pm 45 and $\pm 90^{\circ}$ to the adapted directions. This same set of data also was collected after adaptation. These data allowed us to compare the transfer of adap- 
tation to neighboring saccades with other vectors (i.e., the adaptation field) (Frens and van Opstal, 1994; Noto et al., 1999) in both the electrically induced and visually induced behavioral adaptations.

In five experiments, we presented simple target steps repeatedly in both adapted directions to compare the recovery of saccade amplitude in both stimulation and behavioral experiments.

In two experiments, we used the same stimulus conditions but kept the target illuminated. As the stimulus error vector drove saccade amplitude changes, the visual error was in the opposite direction. Therefore, we expected that this conflict would cause the amplitude change to be less and take longer than if the target were turned off.

Stimulation to change saccade direction (cross-axis adaptation). After we had determined that subthreshold SC stimulation could adapt saccade amplitude, we asked whether it also could alter saccade direction. In these experiments, the monkey made saccades to 10 or $15^{\circ}$ target steps orthogonal to the electrically induced error vector. Both the electrically induced and visually induced adaptations were expected to deviate the saccades in the direction of the error vector. We used a subthreshold $100 \mathrm{~ms}$ pulse train delivered the same way as we induced the amplitude change. During behavioral adaptation, we generated the orthogonal visual error by stepping the target perpendicular to the initial target step. We monitored and adjusted the second target step on-line so that the average visual error was always equal to the putative error vector revealed by suprathreshold SC stimulation.

The effect of different stimulus delays on adaptation. Finally, we tested whether the timing of the SC stimulus train was critical for the amount or time course of adaptation. Two observations suggest that it might be. First, CS activity changes $\sim 80 \mathrm{~ms}$ after the end of saccades for most $\mathrm{P}$ cells (Soetedjo and Fuchs, 2006; Soetedjo et al., 2008). Second, behavioral adaptation experiments suggested that a visual error was most effective if it was present within $100 \mathrm{~ms}$ after the end of the saccade (Shafer et al., 2000). To assess the effect of timing, we used stimulus delays of 0,200 , 400 , and $600 \mathrm{~ms}$. After we had gathered $\sim 300-400$ trials at one of those delays, we collected an additional $\sim 300-400$ saccades at a delay of $80 \mathrm{~ms}$. Except for the experiment with the $600 \mathrm{~ms}$ delay, we used stimulus train durations between 5 and $10 \mathrm{~ms}$ (4-7 pulses) so that the trains did not overlap. We performed 3 experiments for each delay for a total of 12 experiments. If a stimulation delay of $80 \mathrm{~ms}$ is the most effective at producing adaptation, we expected that switching to an $80 \mathrm{~ms}$ delay after having adapted with another delay should produce an additional robust change of saccade amplitude.

Data analysis. During the experiment, we performed on-line analysis to count the number of saccades that was collected, monitor the progression of adaptation, determine the error vector produced by suprathreshold SC stimulation, and keep the adaptation stimulus subthreshold for saccade generation. The on-line analysis detected a saccade when the vector eye movement peak velocity exceeded $75 \%$, and its onset and end were the times when it first exceeded and then fell below $20 \%$ s.

Complete analyses were done off-line from the raw recorded data using two homemade computer programs. First, we processed the eye position traces with an $80 \mathrm{~Hz}$ low-pass 55-coefficient finite impulse response filter to further remove the recording noise. Then, we level triggered on the analog stimulation pulses to transform them into timestamped events. The first program located initial target steps by detecting a $200 \%$ s change in target velocity. Once it found a target step, the program searched between the next 60 and $600 \mathrm{~ms}$ for a vector eye velocity $>75 \% \mathrm{~s}$ and identified that movement as a saccade. The first of the component eye velocities to rise above and the last to fall below $20 \%$ s were taken as the beginning and end of the saccade, respectively. The program measured the amplitude of the target step and the metrics of the detected saccades, as well as saccade reaction times, stimulation latencies, and saccade end times. All of these attributes were saved in a text file with traces of the target and both components of eye position and velocity in a MATLAB (The MathWorks) format file.

The second program read both the attribute text and trace files and first removed trials in which the monkeys did not behave properly. To ensure that the monkeys fixated the target before making a saccade, we required that both the horizontal and vertical eye position were within $\pm 1.5^{\circ}$ of the target before it stepped. We also eliminated saccade trials that were contaminated by blinks. These two criteria eliminated $<10 \%$ of the trials.

The progression of either saccade amplitude or directional changes induced by either SC stimulation or behavioral adaptation was characterized with either an exponential or a linear function, depending on which produced a lower sum-squared error. The percentage of amplitude change that occurred after $N$ trials was calculated as follows:

$$
\begin{aligned}
& \% \text { Amplitude change } \\
& =\frac{\text { mean ampitude }_{\text {trials } N \text { to } N+29}-\text { mean ampitude }_{\text {trials 1-30 }}}{\text { mean ampitude }_{\text {trials 1-30 }}} \times 100 \text {. }
\end{aligned}
$$

The percentage transfer to saccades of different amplitudes and directions than those of the adapted saccade was determined as follows:

\section{$\%$ Transfer}

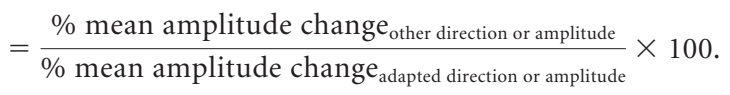

A stimulus- or behavior-induced adaptation was considered successful if the unpaired two-tailed Student's $t$ test of the means of the last 30 and first 30 saccades were significantly different. To evaluate the significance of changes of saccade amplitude or direction across experiments, we used either a nonparametric Wilcoxon signed-rank test for a single sample or a nonparametric Wilcoxon-Mann-Whitney rank sum test for two independent samples. We also used a one-way ANOVA to compare the significance of the change produced by the $80 \mathrm{~ms}$ delay to the changes produced by other delays. We considered a difference to be significant at $p<0.05$.

All experiments were performed in accordance with the recommendations of the National Research Council (1997-2003) and the Society for Neuroscience and exceeded the minimal requirements recommended by the Institute of Laboratory Animal Resources and the Association for Assessment and Accreditation of Laboratory Animal Care International. All procedures were evaluated and approved by the local Animal Care and Use Committee of the University of Washington.

\section{Results}

In these SC stimulation experiments, we gathered 18 saccade amplitude decrease and 17 amplitude increase data sets. Figure 2, $A$ and $B$, illustrates a representative experiment in which the estimated error vector was $2.6^{\circ}$ in amplitude and in the $150^{\circ}$ direction (inset) on the basis of the saccade evoked by suprathreshold stimulation. Subthreshold stimulation after every $15^{\circ}$ saccade directed opposite to the error vector gradually caused a decrease in saccade size (Fig. 2A, open circles) of $2.7^{\circ}$ after 669 saccades. Subthreshold stimulation after every $15^{\circ}$ saccade directed along the error vector gradually caused an increase of $1.2^{\circ}$ after 450 saccades (Fig. $2 \mathrm{~B}$ ). When, $2 \mathrm{~d}$ later, $15^{\circ}$ saccades opposite to and in the direction of this error vector instead were accompanied by an intrasaccadic visual target displacement of $2.6^{\circ}$ at $150^{\circ}$, there was a decrease of $3.8^{\circ}$ after 669 trials and an increase of $1.2^{\circ}$ after 450 trials (gray filled circles), respectively. For this and 17 other stimulation experiments at other sites, the gain decreases from the first to the last 30 saccades of adaptation were significant (Fig. $2 C$, entire adaptation course shown as solid fits). Eighteen error vector-matched behavior experiments (Fig. 2C, dashed fits) showed a significantly greater average reduction, $28.9 \pm 8.5 \%$ versus $19.9 \pm 8.5 \%$. Most plots of the course of each stimulation experiment against its corresponding behavior experiment (from Fig. 2C) lay completely above the line of slope -1 (Fig. 2E), indicating that visually induced was usually more effective than electrically induced adaptation. For those plots, the median of slopes of lines between their start $(1,1)$ and end (i.e., -0.72 ) was significantly greater than -1.0 (Wilcoxon's sign rank test). 
We collected 17 electrically induced amplitude increase adaptations and their error vector matched visually induced adaptations. Based on the first and last 30 adapted saccades, the increase was significant in only 10 of 16 stimulation experiments (range, 6.3-15.6\%), but in 15 of 16 behavior experiments (range, 3.1-18.8\%). For the 10 stimulation experiments with significant increases, the fits of the course of each plotted against that of its error vector matched behavior experiment (from Fig. $2 D$ ) were approximately equally distributed around the line of slope 1 (Fig. 2 F). For those 10, the median slope of lines between their start $(1,1)$ and end was 0.96 , suggesting that, on average, both adaptation paradigms were similarly effective.

\section{Effect of stimulation at different SC sites}

Because the population CS activity of $\mathrm{P}$ cells is greater for small than large saccade dysmetrias (Soetedjo et al., 2008), we explored whether adaptation caused by stimulation at small error sites differed from that caused by stimulation at large error sites. Figure $3, A$ and $B$, shows that stimulation at a $1.1^{\circ}$ error site (circles) was more effective at inducing both amplitude decrease $(A)$ and increase $(B)$ adaptation than was stimulation at a $7.6^{\circ}$ site. For all the amplitude decrease experiments, the percentage change was inversely correlated with error size (Fig. 3C, open circles and solid line). In contrast, the percentage amplitude decrease of the error vectormatched behavior data (black dots) was not correlated with error size; most data clustered between 11 and 26\%.

For the amplitude increase experiments, the electrically induced percentage change for errors $<2.7^{\circ}$ was similar (median, $8.68 \% ; n=7$ ), but for larger errors ranged from 0 to $11 \%$ (median, 3.23\%; $n=10$ ) (Fig. 3D). Therefore, stimulation of sites signaling larger errors could cause a variety of amplitude increases. Again, visually induced amplitude increases were unrelated to error size.

In summary, the size of the stimulusevoked error vector had a consistent negative effect on the magnitude of amplitude decreases but a variable influence on amplitude increases. In contrast, different sizes of matched visual error vectors had no effect on the magnitude of either amplitude decrease or increase behavioral adaptations.

\section{Characteristics of SC electrically induced amplitude adaptation}

Electrically induced adaptation fields

The effect of behavioral adaptation of saccades transfers less and less as the tested saccades deviate more and more from the
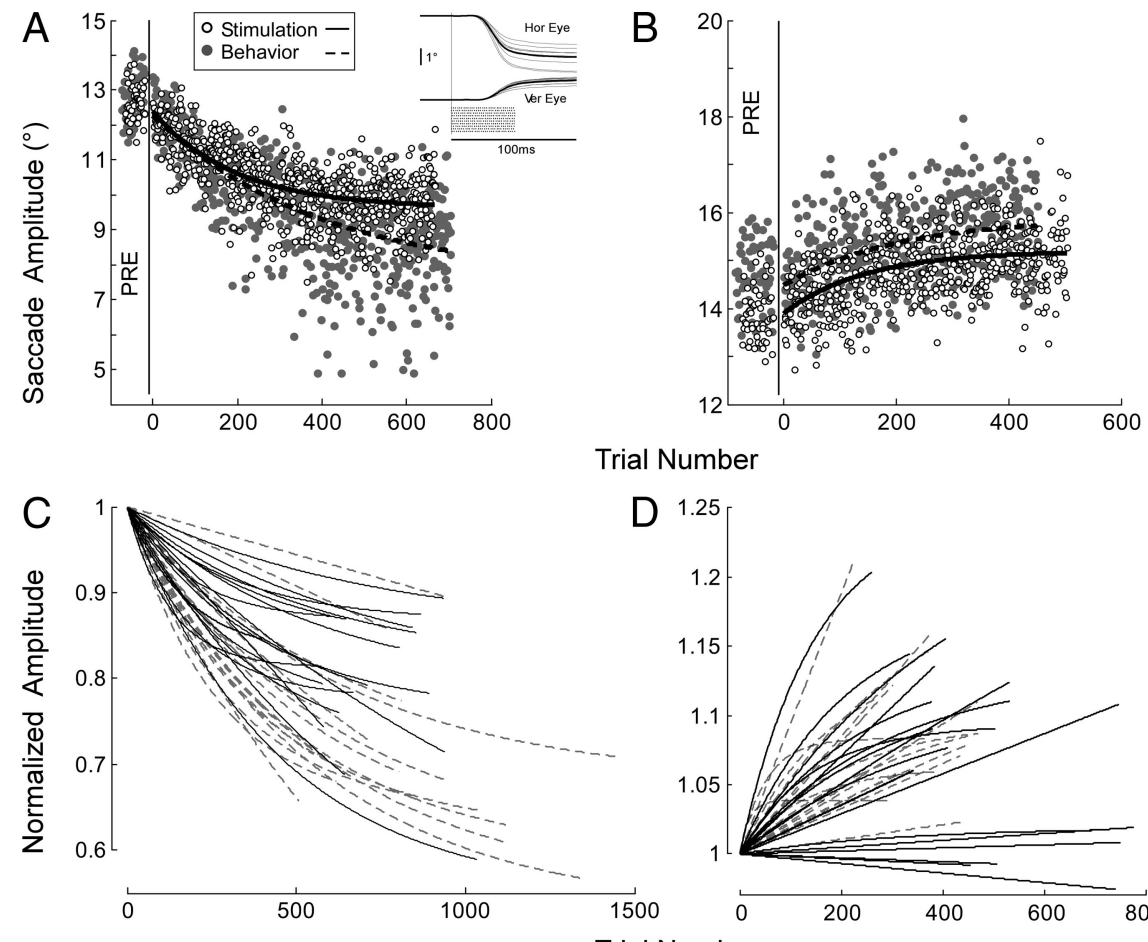

Trial Number

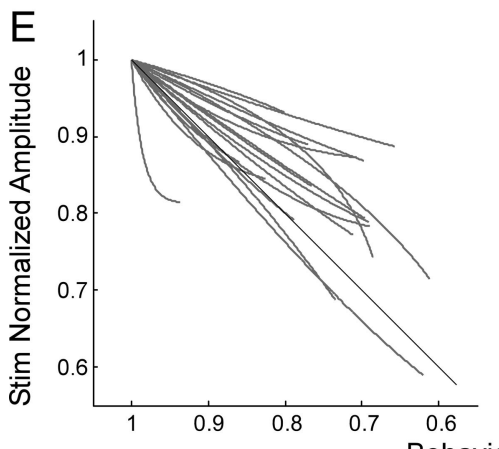

Trial Number
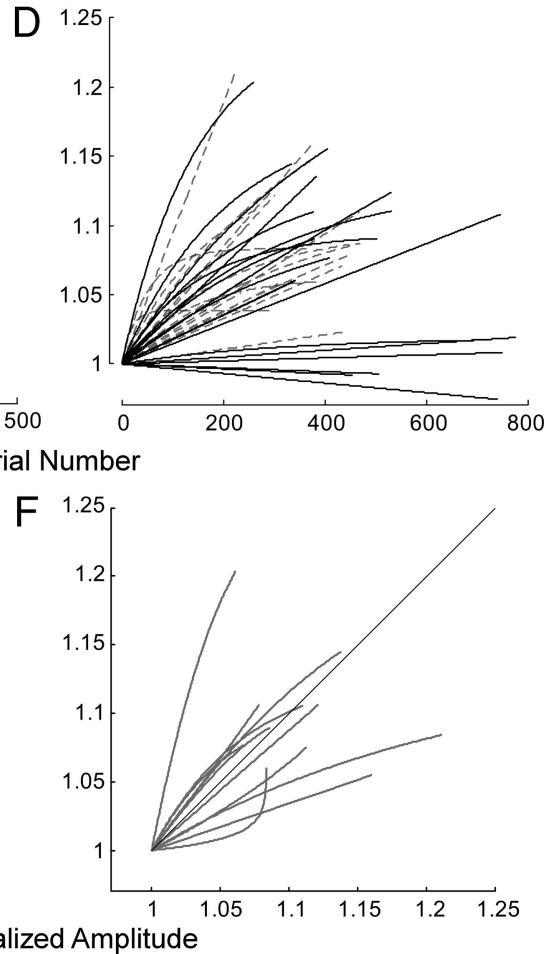

Figure 2. Comparison between electrically induced and visually induced adaptation. $A, B$, Saccade amplitude as a function of sequential trial number during electrically induced (circles) and error vector matched visually induced (gray dots) adaptations. Suprathreshold stimulation at this $\mathrm{SC}$ site evoked saccades of $2.6^{\circ}$ amplitude at an angle of $150^{\circ}(\boldsymbol{A}$, inset; thick curves are mean eye positions), which we consider to be an estimate of the putative error vector. We produced stimulus-induced adaptation with a subthreshold stimulus train $(200 \mathrm{~Hz} ; 100 \mathrm{~ms} ; 11 \mu \mathrm{A})$ beginning $80 \mathrm{~ms}$ after the saccade landed. Saccades to $15^{\circ}$ target steps in the opposite direction $(\boldsymbol{A})$ decreased their amplitudes; those in the same direction $(\boldsymbol{B})$ increased their amplitudes. Exponential fits for the electrically and visually induced adaptations are solid and dashed, respectively. Here and in Figures 3 and 7 , data before trial number zero (PRE) were collected before adaptation. $\boldsymbol{C}, \boldsymbol{D}$, For all neurons, similar fits of amplitude decrease $(\boldsymbol{C})$ and increase (D) adaptations induced either by an electrical (solid curves) or visual stimulus (dashed curves). Each fit is normalized to start at 1 by dividing all saccade amplitudes during adaptation by the value of the fit at trial $1 . E, F$, Fits of electrically induced adaptations $(\boldsymbol{C}, \boldsymbol{D}$, solid curves) versus those of error-matched visually induced adaptations ( $\boldsymbol{C}, \boldsymbol{D}$, dashed curves), plotted only up to the smaller number of trials in the pair. A pair of adaptations with identical time courses would yield a line with a slope of -1 or 1 (black lines) for an amplitude decrease $(\boldsymbol{E})$ or increase $(\boldsymbol{F})$, respectively.

adapted vector. Plots of the amount of amplitude adaptation as a function of the amplitude and direction of the initial target step produce the adaptation field (Frens and van Opstal, 1994; Noto et al., 1999). We asked whether electrically induced adaptation also exhibited such an adaptation field.

In the electrically induced adaptation experiment shown in Figure 2, $A$ and $B$, saccades to $15^{\circ}$ target steps along 330 and $150^{\circ}$ underwent significant amplitude decrease and increase adaptation, respectively. These adaptations transferred to saccades elicited by target steps with different directions (Fig. 4A) and amplitudes (Fig. $4 B)$. Saccades directed along 15 and $105^{\circ}\left(45^{\circ}\right.$ 
A

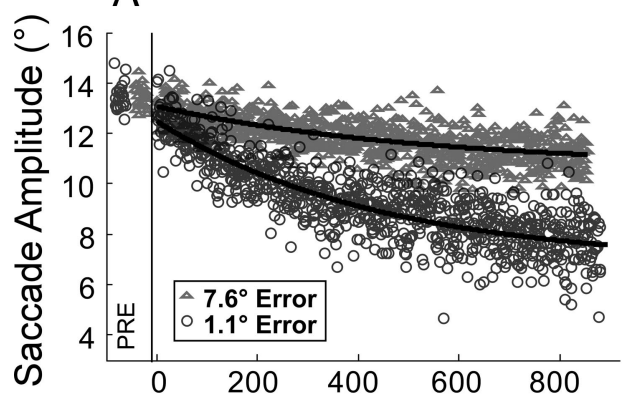

B

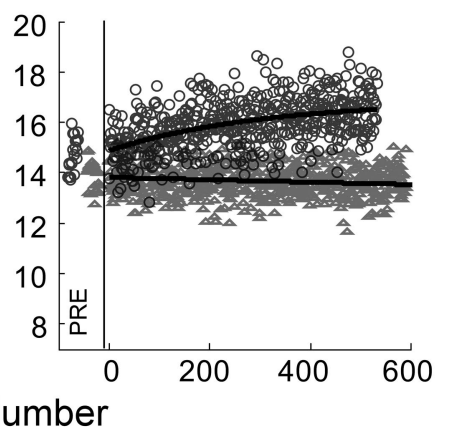

Trial Number

C

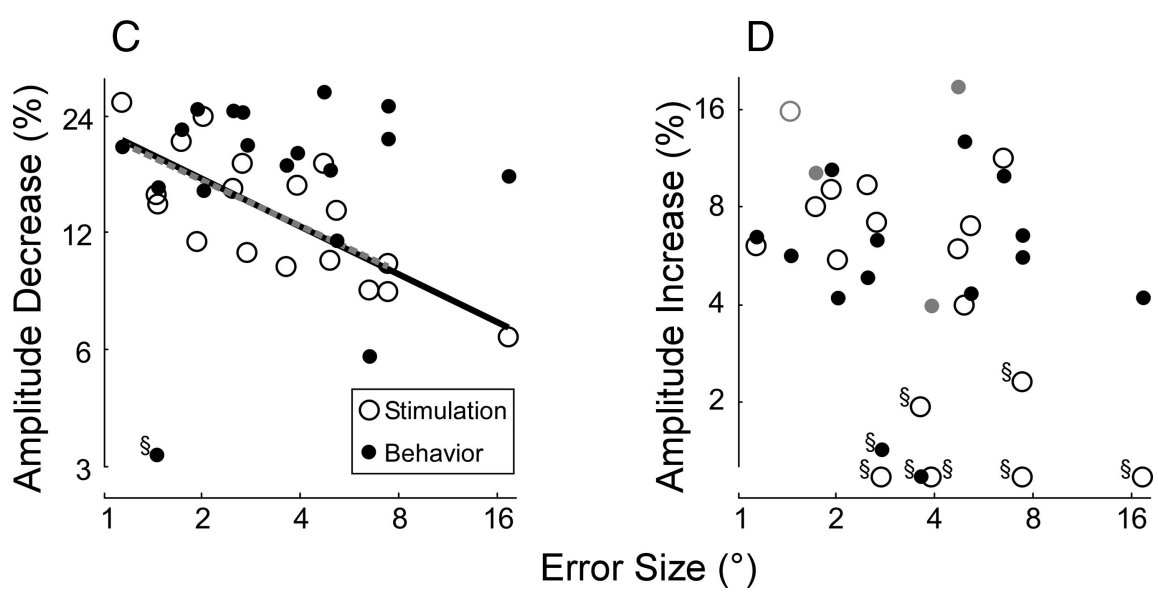

Figure 3. Saccade adaptation produced by stimulation at different $S C$ sites. $\boldsymbol{A}, \boldsymbol{B}$, Courses of amplitude decrease $(\boldsymbol{A})$ and increase $(\boldsymbol{B})$ adaptation induced by stimulation of $7.6^{\circ}$ (triangles) and $1.1^{\circ}$ (circles) error sites in separate experiments. Three fits are exponential and one is linear. $\boldsymbol{C}, \boldsymbol{D}$, Log percentage amplitude decrease or increase, respectively, caused by electrically induced (open circles) and visually induced adaptation (dots) as a function of log error size. Logs are used because the distribution of error sizes was skewed toward large errors. Percentage amplitude decrease was computed after 373 trials. Percentage amplitude increase was computed after 270 trials, except in four experiments ( $\boldsymbol{D}$, gray circle and dots) for which trials ranged from 206 to 246. In $C$, the solid line is the linear regression of the stimulation data $(r=0.76 ; n=18)$; the regression was not significantly different after the largest error size datum $\left(17.2^{\circ}\right)$ was removed (dashed line). In $\boldsymbol{D}$, § identifies percentage amplitude increases that were not significantly different from zero ( $p>0.05$, two-tailed Student's $t$ test).

from the adapted directions) also exhibited significant (Fig. $4 A$, asterisks) decreases and increases, respectively $(p<0.05$, two-tailed Student's $t$ test). In other directions, adaptation transfer was not significant. Along $330^{\circ}$, reduction of the adapted saccades to $15^{\circ}$ targets significantly reduced saccades to both 10 and $20^{\circ}$ targets (Fig. $4 \mathrm{~B}$, asterisks; positive vector amplitudes). Along $150^{\circ}$, increase of the adapted saccade to $15^{\circ}$ targets transferred significantly only to saccades to $20^{\circ}$ targets (Fig. $4 B$, asterisks; negative vector amplitudes). The average transfer data for this experiment are shown in Figure 4, $C$ and $D$ (red), where $0^{\circ}$ indicates the adapted direction and $15^{\circ}$ the adapted size. Data for the other 12 experiments that tested electrically induced adaptation fields after amplitude decreases (solid gray lines) and increases (dashed lines) are shown in the same format.

We tested adaptation fields in 13 of 18 amplitude change experiments. All 13 amplitude reduction experiments (Fig. 4C,D, solid gray lines) showed qualitatively similar direction and amplitude adaptation fields. The median reduction (bold symbols) at the adapted direction $\left(0^{\circ}\right)$ and amplitude $\left(15^{\circ}\right)$ showed a symmetrical median transfer of 26.3 and $23 \%$ to angles $\pm 45^{\circ}$ away (Fig. 4C) and an asymmetric transfer that favored larger saccades (Fig. $4 D$ ) [median transfers of $85.3 \%$ to $20^{\circ}$ targets, $57 \%$ to $10^{\circ}$, and $24.8 \%$ to $5^{\circ}$ (NS, not significant transfer)]. In the $\pm 90^{\circ}$ directions, the median changes were NS.
In the direction expected to produce electrically induced amplitude increases, only 8 of 13 experiments showed a significant change. In those 8 , there was a small median transfer (6.2 and $22.2 \%$; both NS) to angles $\pm 45^{\circ}$ away and an asymmetric transfer that favored larger saccades (median transfers of $80.2 \%$ to $20^{\circ}, 41 \%$ to $10^{\circ}$, and $11 \%$ to $\left.5^{\circ}\right)$. For $\pm 90^{\circ}$, no experiments showed a significant amplitude increase. Finally, the shapes of individual adaptation fields showed more variation than those after amplitude reductions (Fig. $4 C, D$, dashed vs solid gray lines).

As a comparison, Figure $4, E$ and $F$, shows the direction and amplitude adaptation fields produced in visually induced adaptation experiments with a similar range of error vectors. The median transfers of adaptation decreases to +45 and $-45^{\circ}$ were 30.9 and $26.7 \%$, and to +90 and $-90^{\circ}$ were $7.1 \%$ (NS) and $5.6 \%$, respectively $(n=11)$ (Fig. $4 E$ ); they were $79.4,52$, and $28.6 \%$ to saccades to 20,10 , and $5^{\circ}$ target steps, respectively $(n=11)$ (Fig. $4 F$ ). The median transfers of adaptation increases to +45 and $-45^{\circ}$ were 0 and $38.1 \%$, respectively, and zero to $\pm 90^{\circ}$ $(n=8)$ (Fig. $4 E$ ). The median transfers of increases to 20,15 , and $5^{\circ}$ target steps were 88.3, 35.7, and 24.3 (NS), respectively $(n=7)$ (Fig. 4F).

The medians of the percentage amplitude change produced by electrically induced adaptation captured the essential characteristics of the visually induced adaptation fields quite well. In particular, the transfer to larger saccade amplitudes was stronger for both amplitude increase and decrease adaptations (Fig. $4 D, F$ ). Also, there was essentially no transfer of adaptation to saccades orthogonal to the adapted direction (Fig. $4 C, E)$. The only quantitative difference was that visually induced adaptation at the adapted direction and amplitude was significantly greater (Fig. 4C-F, bold circles) for amplitude decreases, but not for amplitude increases (bold squares), than electrically induced adaptation.

\section{Electrically induced adaptation retention and recovery}

If the electrically induced amplitude change is a genuine form of motor learning, it should show retention and recovery (or extinction) when the stimulation is discontinued. We tested the retention and time course of extinction in five amplitude decrease and two amplitude increase stimulation and error vector-matched behavioral experiments. All began with an electrically or visually induced increase or decrease in saccade amplitude.

In the experiment illustrated in Figure $5 A$, the average saccade amplitude to $10^{\circ}$ target steps started at $8.4^{\circ}$ (gray squares, solid red curve) and decreased to $6.4^{\circ}$ after 627 trials. When stimulation was then discontinued and the monkey tracked ordinary $10^{\circ}$ target steps, saccade amplitude initially jumped by $\sim 0.4^{\circ}$ toward its preadapted value and then gradually recovered all the way. The error vector-matched behavioral adaptation (black dots, dashed red curves) showed a similar amplitude jump $\left(0.6^{\circ}\right)$ 
at the start of recovery and a gradual return toward the preadapted amplitude.

In the experiment illustrated in Figure $5 B$, average saccade amplitude increased gradually from 10.5 to $11.4^{\circ}$ (gray circles, solid green curve) after 381 trials. When stimulation was discontinued and the monkey tracked ordinary $10^{\circ}$ target steps, saccade amplitude decreased suddenly by $\sim 0.4^{\circ}$ and then recovered gradually to preadapted amplitudes. Again, the error vector-matched behavioral adaptation and its recovery resembled those after SC stimulation.

Figure $5 C$ shows the fitted curves for all electrically induced adaptations (solid lines) and their error vector-matched behavioral counterparts (dashed lines) in matching colors. Visually induced behavioral adaptation occurred faster than electrically induced adaptation in three of five amplitude decrease experiments and in both amplitude increase experiments. However, behavioral recovery was much slower in all seven. The amplitude jumps at the beginning of recovery after electrically induced and visually induced adaptations were not statistically different. In summary, electrically induced adaptations exhibit both retention and extinction, which qualitatively resemble those of visually induced adaptation. Electrically induced adaptation fades more rapidly.

\section{Characteristics of SC electrically induced direction adaptation}

Can the error vector signaled by SC stimulation drive changes in saccade direction as well as amplitude? To test this, we stimulated after saccades that were perpendicular to the error vector. In the example illustrated in Figure 6A-D, suprathreshold SC stimulation had elicited a $1^{\circ}$ leftward saccade. We expected that subthreshold stimulation delivered after either upward or downward saccades would gradually cause them to veer to the left toward angles $>90$ and $<270^{\circ}$, respectively. This indeed happened after repeated stimulations over 600-800 trials (Fig. 6A, $B$, black circles). Figure $6 C$ compares 20 saccade trajectories early (gray) and late (pink) in adaptation. The companion behavior experiment using the same visual error vector produced similar courses of adaptation (Fig. 6A, B, gray squares, dashed fits) and similar changes in the saccade trajectories (Fig. 6D).

The courses of six stimulation (solid curves) and four error-matched behavioral adaptations (dashed curves with the same colors) are shown in Figure 6E. After 424 stimulation trials (the shortest data set), the median change in upward and downward saccade vector angles was $5.7^{\circ}$ (range,
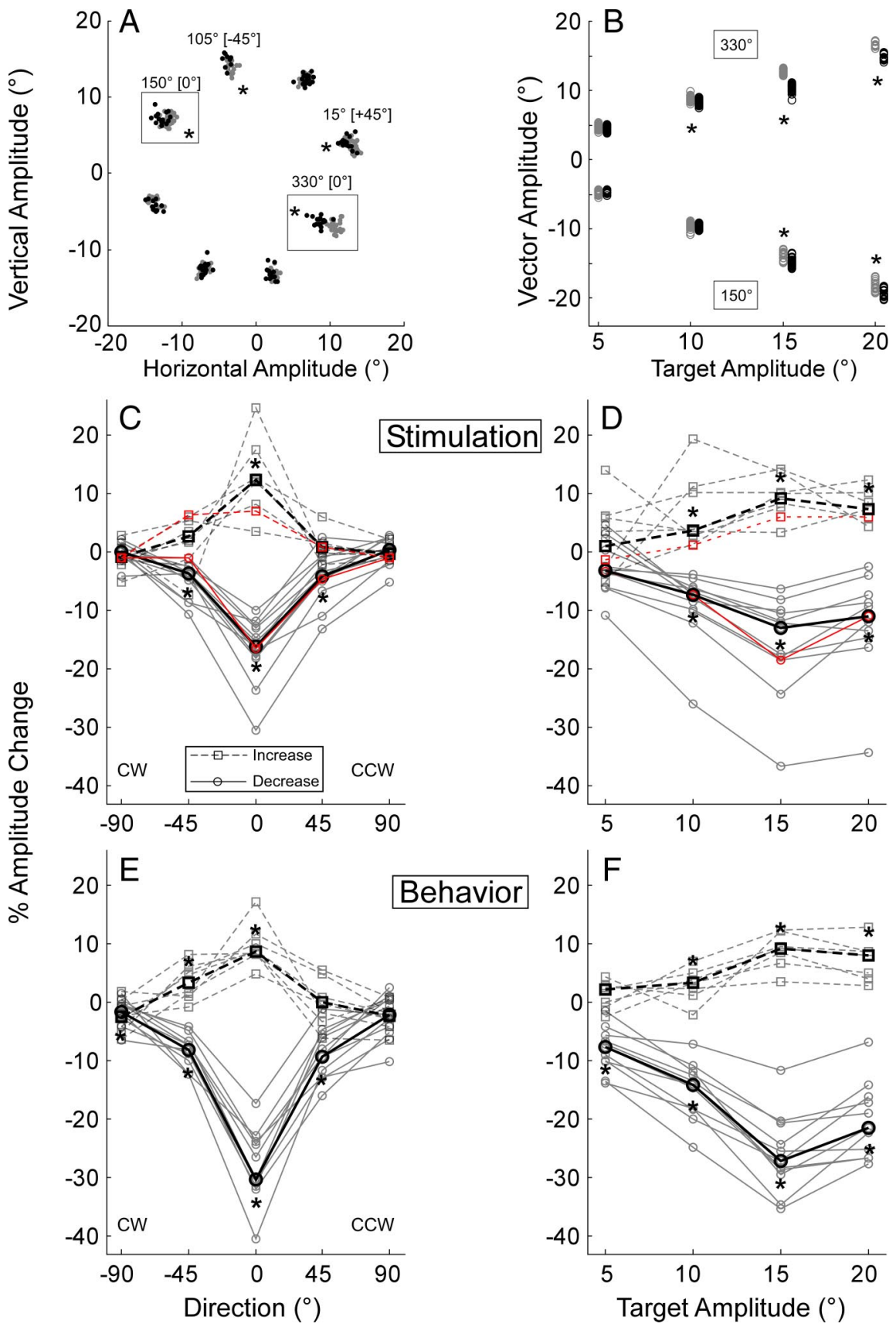

Figure 4. Electrically and visually induced adaptation fields. $\boldsymbol{A}, \boldsymbol{B}$, Vertical and horizontal components of saccades in eight directions $(\boldsymbol{A})$ and vector saccade amplitudes to four target step sizes $(\boldsymbol{B})$ before (gray) and after (black) stimulus-induced adaptation. $C, D$, Percentage amplitude change as a function of saccade direction and amplitude after electrically induced adaptation (gray). Adapted direction is normalized to $0^{\circ}$. For amplitude decreases, a median $-16.14 \%$ amplitude change in the adapted direction $\left(\boldsymbol{C}\right.$, heavy circle at $\left.0^{\circ}\right)$ caused -4.25 and $-3.71 \%$ amplitude changes at $\pm 45^{\circ}$; a median $-12.9 \%$ change at the adapted amplitude $\left(15^{\circ}\right)$ caused -11 and $-7.3 \%$ changes of saccades to 20 and $10^{\circ}$ target steps $(\boldsymbol{D})$. For amplitude increases, a median $+12.21 \%$ amplitude change in the adapted direction caused no significant change at $\pm 45^{\circ}(\mathbf{C})$; a median $+9.1 \%$ change at the adapted amplitude caused +7.3 and $+3.7 \%$ changes of saccades to 20 and $10^{\circ}$ target steps $(\boldsymbol{D})$. $\boldsymbol{E}, \boldsymbol{F}$, Visually induced adaptation fields. For amplitude decreases, a median $-30.31 \%$ amplitude change in the adapted direction caused -9.37 and $-8.1 \%$ changes at $\pm 45^{\circ}$, and -2.15 and $-1.7 \%$ at $\pm 90^{\circ}(\boldsymbol{E}) ;$ a median $-27.1 \%$ adapted amplitude change caused -7.75 , -14.1 , and $-21.5 \%$ changes of saccades to 5,10 , and $20^{\circ}$ target steps, respectively $(\boldsymbol{F})$. For amplitude increases, a median $+8.56 \%$ amplitude change in the adapted direction produced 0 and $+3.3 \%$ changes at $\pm 45^{\circ}(\boldsymbol{E}) ;$ a median $+9.1 \%$ change at the adapted amplitude caused +3.24 and $+8 \%$ changes of saccades to 10 and $20^{\circ}$ target steps $(\boldsymbol{F})$. The asterisks indicate significant changes ( $p<0.05$, Wilcoxon-Mann-Whitney test).

$2.22-7.13)$ and $-4.83^{\circ}(-2.77$ to -8.77$)$, respectively. In four experiments, the angle changes of upward and downward saccades were equal $(p>0.05)$. In the other two experiments, they differed by 3.34 and $3.48^{\circ}$. For behavioral adaptations, the me- 

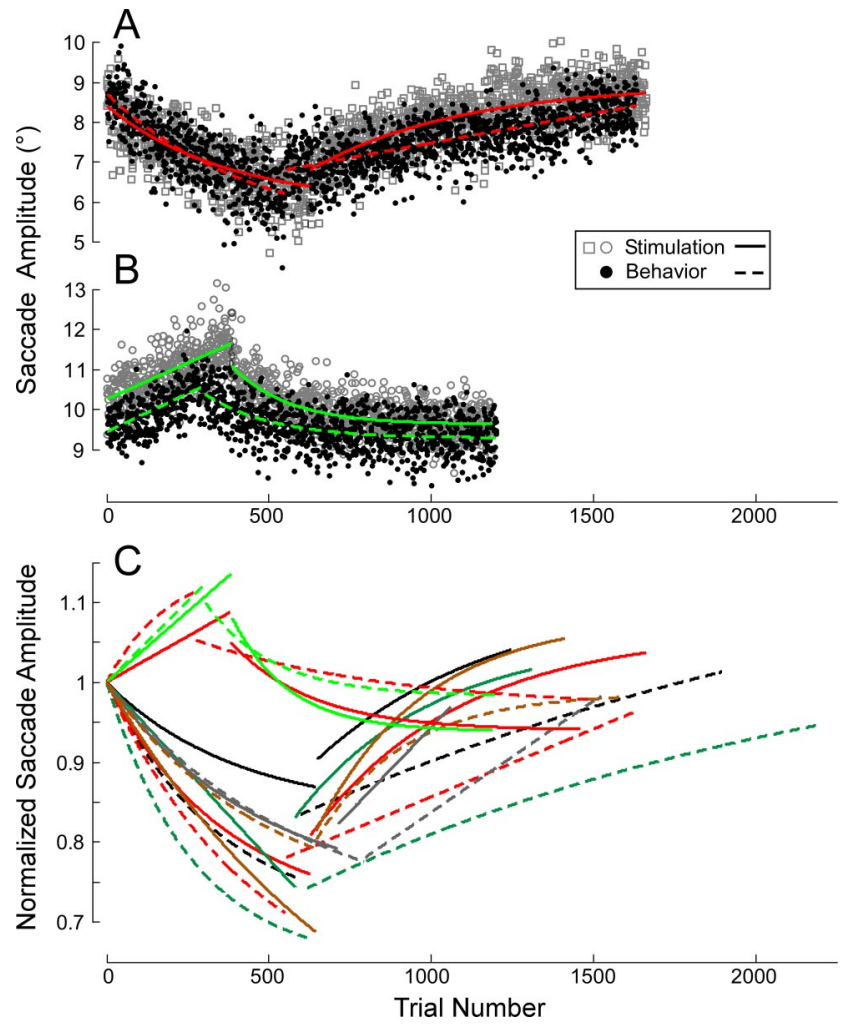

Figure 5. Courses of recovery of electrically induced and visually induced adaptation. $A, B$, Saccade amplitude versus sequential trial number during electrically induced (gray symbols, solid fits) and error vector matched visually induced (black dots, dashed fits) adaptation followed by recovery. C, Summary of seven exponential fits of normalized amplitude as a function of trial number during either electrically induced (solid lines) or error vector-matched (same color) visually induced (dashed lines) adaptation and recovery. Both adaptation and recovery fits were normalized with respect to the value of the fit at trial 1 . The smaller number of amplitude increase trials was a result of the $\pm 20^{\circ}$ limitation in eye position eccentricity.

dian angle changes in the upward and downward directions, $6.72^{\circ}$ (range, $3.6-8.07^{\circ}$ ) and $-5.33^{\circ}$ (range, -4.77 to $-6.94^{\circ}$ ), respectively, were statistically indistinguishable from those produced by stimulation. In two experiments, the angle changes of upward and downward saccades were equal, whereas, in the other two, they differed by 2.1 and $2.81^{\circ}$.

To compare the courses of electrically induced and visually induced adaptations directly, we plotted their fits against each other for both angle increases (Fig. 6F, green curves) and (absolute) decreases (red curves). On average, the slopes of lines between the origin and the last point of the curves were not significantly different from unity (black line; Wilcoxon's sign rank test), suggesting that electrically and visually induced crossaxis adaptation followed very similar courses.

\section{Effects of stimulus timing on adaptation}

In the previous experiments, we stimulated $80 \mathrm{~ms}$ after the end of each saccade when the probability of CS occurrence began to increase (Soetedjo et al., 2008). Here, we tested whether other stimulus delays altered the quality of the adaptation. In four sets of three experiments each, we started with stimulus delays of 0 , 200, 400, or $600 \mathrm{~ms}$. After $\sim 300-400$ trials, we switched the stimulus delay to $80 \mathrm{~ms}$ and collected another $\sim 300-400$ trials. For the experiment illustrated in Figure 7, $A$ and $B$, a 600 ms delay (inset above gray dots) caused a modest amplitude decrease for saccades opposite to the error vector $\left(\sim 0.6^{\circ}\right.$ after 394 trials; gray dots and red fit) but also a slight anomalous decrease in the same direction as the error vector $\left(\sim 0.12^{\circ}\right.$ after 320 trials; gray dots and red fit). Switching to an $80 \mathrm{~ms}$ delay (inset above black dots) enhanced the amplitude decrease ( $A$, black dots and red fit) and produced the expected amplitude increase for saccades in the opposite direction ( $B$, black dots and red fit). Normalized amplitude (use right scales) fits for this experiment and two others (green and blue fits) show similar characteristics.

To summarize, Figure $7 C$ compares the percentage amplitude changes produced by either a $0,200,400$, or $600 \mathrm{~ms}$ delay and the subsequent $80 \mathrm{~ms}$ delay for all 12 experiments. The $80 \mathrm{~ms}$ delay produced a significantly greater change in 11 of 12 amplitude increase experiments (positive percentage, open symbols above line of slope 1.0) and in 9 of 12 amplitude decrease experiments (negative percentage, open symbols below line). Figure $7 D$ illustrates the mean percentage changes for the three amplitude increase and decrease experiments performed at each delay. At delays of 0, 200, 400, and $600 \mathrm{~ms}$ (filled circles), all experiments but one ${ }^{*}$ ) showed a nonsignificant amplitude increase. The grand averages (green) for those delays were not significantly different. In contrast, all three of the $80 \mathrm{~ms}$ delay experiments produced significant amplitude increases (average, 7.7\%; range, $7.1-8.3 \%)$.

When stimulation was expected to cause amplitude decreases, a $0 \mathrm{~ms}$ delay never produced a significant change, but delays of 200, 400, and $600 \mathrm{~ms}$ all did. Grand averages of data at these longer delays (red filled squares) were not significantly different, but were significantly less than those produced with the $80 \mathrm{~ms}$ delay and significantly greater than those with the $0 \mathrm{~ms}$ delay (one-way ANOVA, post hoc with Bonferroni's correction). The delay of $80 \mathrm{~ms}$ produced an average 17\% (range, 11-20\%) amplitude decrease. Therefore, the $80 \mathrm{~ms}$ stimulus delay was the most effective in inducing either amplitude increase or decrease adaptation.

\section{Interaction with behavioral adaptation}

In two experiments, we delivered the stimulus without turning off the target to evaluate whether the presence of a conflicting visual error reduces the effect of the surrogate electrically induced error. Electrically induced adaptation caused saccades to become either hypometric or hypermetric, so the visual error driving adaptation was in the opposite direction. We compared the course of amplitude changes in 2 such "conflict" experiments (error vectors of 1.4 and $2.1^{\circ}$ ) with the average of those experiments with similar error vectors (range, $1.1-2.6^{\circ}$ ) in which the occurrence of saccades extinguished their target as usual. In the conflict condition, the amplitude increases of 2.3 and 2.4\% (299 trials) were significantly smaller than the median produced by six electrically induced adaptations with the target off (Fig. 3D, six circles at errors $<2.7^{\circ}$; median, $8.9 \%$ ). In the conflict condition, the amplitude reductions of 12.4 and 9.9\% (402 trials) were significantly less than the average produced by seven electrically induced adaptations with the target off (Fig. $3 C$, seven circles at errors $<2.7^{\circ}$; median, $18.1 \%$ ). Therefore, a conflicting visual error reduces an electrically induced amplitude change.

\section{Discussion}

Our results show that subthreshold stimulation of the SC after each targeting saccade produces gradual changes of saccade amplitude or direction. These stimulus-evoked changes display four salient features that strongly resemble those of behavioral saccade adaptation induced with matching visual vector errors. First, if saccades are followed by stimulus-evoked or visual errors in the same direction, they grow larger. Saccades in the opposite 
direction to the errors shrink (Fig. 2). Saccades in directions orthogonal to either the electrically induced or visual errors gradually veer toward the error direction (Fig. 6). Second, changes in amplitude or direction occur slowly over hundreds of trials. They show a sudden jump at the onset of extinction and recover slowly after either the stimulus or visual error signal is discontinued. Third, both electrically and visually induced adaptations that decrease amplitude are larger and occur faster than adaptations that increase amplitude (Fig. 5) (Straube et al., 1997). Fourth, changes produced by both electrically induced and visual errors exhibit adaptation fields in which transfer from the adapted saccade to larger saccades is greatest, and transfer to saccades in directions orthogonal to the adapted saccades does not occur (Fig. 4) (Frens and van Opstal, 1994; Noto et al., 1999). Considering how artificial SC stimulation is, these qualitative similarities are remarkable.

Moreover, the characteristics of electrically induced adaptation reflect the properties of CS activity. First, the stimulus delay of $80 \mathrm{~ms}$, which produces the greatest amplitude decrease and increase adaptation (Fig. 7), is about the average latency of the most vigorous CS activity (Soetedjo et al., 2008). Previous studies have shown that behavioral adaptation is most effective with a visual error delay between $\sim 0$ and 60 ms (Shafer et al., 2000). Because the visual latency of $\mathrm{SC}$ neurons is $\sim 60-70 \mathrm{~ms}$ (Goldberg and Wurtz, 1972; Mays and Sparks, 1980), the error signal leaves the SC between 60 and $130 \mathrm{~ms}$ after the saccade, an interval that includes our most effective stimulus delay. Second, stimulation at small error sites in the SC generally produces greater adaptation than stimulation at larger error sites (Fig. 3). This result would be expected if adaptation were driven by the population CS activity, which is larger for small than large errors (Soetedjo et al., 2008). However, visually induced adaptation shows no such error dependence (Fig. $3 C, D$ ). In contrast, Robinson et al. (2003) concluded that error size does affect amplitude reduction adaptation. However, if one considers only errors of $\leq 8^{\circ}$, like those in the majority of our behavioral experiments (Fig. 3), adaptation of both their 12 and $18^{\circ}$ saccades also exhibited no error dependence over that range [Robinson et al. (2003), their Fig. 4A]. The remarkable similarities in electrically and visually induced adaptation together with the reflection in electrically induced adaptation of the sensitivity of CS occurrence to the timing and size of saccade errors lead us to conclude that stimulation of the SC produces a surrogate error signal that drives saccade adaptation through the CS pathway. As we mention in Introduction, there is a plausible anatomical substrate for such a pathway because the SC projects strongly to the contralateral b-nucleus of the medial accessory olive (MAO) (Huerta and Harting, 1984), which, in turn, projects to the contralateral oculomotor vermis, lobules VIc and VII (Kralj-Hans et al., 2007). In patients, interference with this pathway compromises behaviorinduced adaptation (Waespe and Baumgartner, 1992; Helmchen et al., 1994). Our observation that electrically induced adaptation is reduced when the target is left on to cause a conflicting visual error signal also is consistent with, but not proof that, the two error signals share a common pathway. To prove the link between SC stimulation and adaptation, we must record from a single oculomotor vermis $\mathrm{P}$ cell and simultaneously stimulate the SC to drive both CSs and adaptation. Finally, our subthreshold stimulation rarely evoked saccades, so adaptation cannot have been caused by motor signal.

If the error signals of both adaptation paradigms were routed through the same pathway, why did most electrically induced adaptations develop more slowly and recover more rapidly? Perhaps our stimulation activated a smaller area of the SC than would a visual error during behavioral adaptation. A visual error of $2.5^{\circ}$ activates an area with a diameter of at least $1.5 \mathrm{~mm}$ (Anderson et al., 1998), whereas we estimate that our average stimulus of 18 $\mu$ A would spread to a distance of $<250 \mu \mathrm{m}$ (Abzug et al., 1974). If activation of more SC tissue activates more inferior olive neurons and thereby drives more CSs, perhaps adaptation would be faster and stronger and thus take longer to dissipate. We think that the error signal originates from the intermediate layer of the 

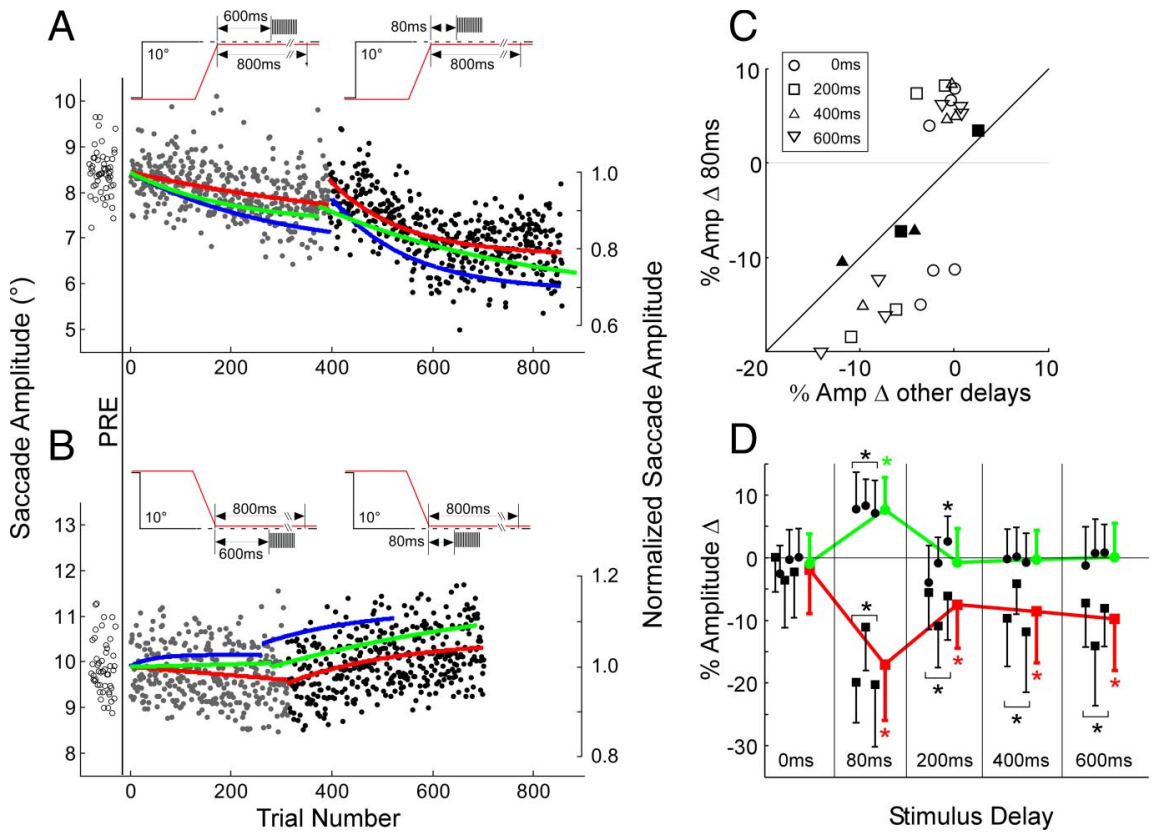

Figure 7. Effect of stimulus delay on adaptation. $\boldsymbol{A}, \boldsymbol{B}$, Saccade amplitude as a function of sequential trial number for three different experiments. In $\boldsymbol{A}$ and $\boldsymbol{B}$, the electrically induced error vector should have produced a decrease and increase, respectively, in saccade amplitude. The stimulus delay began $600 \mathrm{~ms}$ after each saccade (inset, gray dots and curves from 0 to $\sim 300-400$ trials) and then was switched to $80 \mathrm{~ms}$ (inset, black dots, and curves). The red curves are fits of raw amplitude data (dots) of one of the three experiments. The green and blue curves are fits from the other two experiments (raw data not shown). Fits are either linear or exponential. To compare fits across three experiments, all fits are normalized to the fitted amplitude of trial 1 (right scales). C, Percentage amplitude changes $(\Delta)$ produced by the later 80 ms versus the earlier $0,200,400$, or 600 ms delays of all 12 experiments like those in $\boldsymbol{A}$ and $\boldsymbol{B}$. For amplitude decreases and increases, changes were measured after 373 and 262 trials (Eq. 1), respectively. Nonsignificant differences lie near the line of slope 1.0 (filled symbols, $p>0.05$, two-tailed Student's $t$ test). D, Comparison of percentage amplitude changes produced by $0,80,200,400$, and 600 ms stimulus delays for both amplitude increase (circles) and decrease (squares) adaptation. For each delay, there were three experiments with the same primary saccade size $\left(10^{\circ}\right)$ and comparable error vector sizes (range, $0.6-3.5^{\circ}$; mean, $2.3 \pm 0.87^{\circ}$ ). The red and green symbols are mean percentage amplitude changes of the three experiments within the same delay bin. The asterisks identify percentage amplitude changes that are significantly different from zero ( $p<0.05$, one-sample two-tailed Student's $t$ test). Error bars indicate SD.

SC where cells respond to both visual targets and the saccades that they evoke (Mays and Sparks, 1980). Cells in this layer are retrogradely labeled after MAO injections of HRP (Huerta and Harting, 1984). Because of this labeling study, we always attempted to place our stimulating electrodes in the intermediate layer and did not systematically investigate whether stimulation in the deep and superficial layers was less effective.

In addition to the possible differences in the size of the SC area that is active during electrically and visually induced adaptations, there are two other possible explanations for the difference in the robustness of the two kinds of adaptation. First, as we mention in Introduction, an attractive hypothesis (Marr, 1969; Albus, 1971) suggests that errors produced by persistent saccade dysmetrias gradually change the probability of CS occurrence, which by some synaptic mechanism, alters the activity of simple spikes driven by (saccade-related) mossy fiber inputs to the same P cell. Perhaps our subthreshold electrical activation of the SC did not produce its normal input to the nucleus reticularis tegmenti pontis (Harting, 1977), a major source of the saccade-related mossy fibers to the oculomotor vermis (Kralj-Hans et al., 2007). Consequently, the putative changes of $\mathrm{P}$-cell excitability attributable to changes in CS activity caused by SC stimulation were altering a weakened mossy fiber input so less adaptation occurred. Second, the SC itself might be an extracerebellar site of plastic changes associated with saccade adaptation (Takeichi et al., 2007). The natural visual error may be more effective at inducing changes of
SC activity during adaptation than is an electrically induced error signal.

In two other studies, brainstem stimulation also caused gradual changes in saccade amplitude. In one, subthreshold stimulation of the SC after horizontal saccades gradually shifted their end points in the direction of the error vector of the stimulated site (Kaku et al., 2009). Their results are consistent with those in our Figure 6, which shows that SC stimulation creates a vector error signal that can adapt the direction as well as the amplitude of saccades. However, they tested no other characteristics of adaptation, such as the adaptation field, adaptation recovery, or the effect of stimulus timing. Nor did they compare their stimulus-induced adaptation to vector error matched behavioral adaptation. Whereas their study indicates that SC stimulation can evoke saccade adaptation, our data, which were collected at the same time, show that such electrically induced adaptation is remarkably similar to the matched visually induced adaptation obtained in the same monkeys. Moreover, our data reflect the constraints imposed by the characteristics of CS error signals (Soetedjo and Fuchs, 2006; Soetedjo et al., 2008).

Others have stimulated an area of the midbrain tegmentum near the oculomotor complex and produced amplitude increase and decrease adaptations that were equally robust (Kojima et al., 2007). These data differ from those in this paper, which showed a much poorer adaptation for amplitude increases. Moreover, their robust adaptations were obtained even when the visual target remained illuminated. Perhaps the differences between their results and ours could be attributed to the existence of multiple adaptation mechanisms.

In conclusion, our data lead us to suggest an additional role for the SC in the neuronal control of saccades: that of the source of the error signal for saccade adaptation. Furthermore, the reflection of the timing and saccade error dependency of CS activity in the characteristics of electrically induced adaptation allows two additional suggestions. First, the CS activity in the oculomotor vermis, which reports the direction and size of the error of dysmetric saccades, originates in the SC and is relayed to the oculomotor vermis through the inferior olive. Second, this CS activity serves as the signal that drives motor learning to reduce that error.

An analogous error signal might also have been revealed for smooth pursuit eye movements. Stimulation of the medial temporal cortex, an area with movement-sensitive cells that project to the nucleus of the optic tract and thence to the inferior olive (Mustari et al., 1994), induces pursuit adaptation (Carey et al., 2005). Similar studies have not been done for other precision movements. If the cerebellum indeed plays a unitary role in the motor learning of all types of movement, we suggest that CS activity in parts of the cerebellar vermis involved with those movements (e.g., finger pointing) could be the source of the motor error signal that drives adaptation of their trajectories as well. 


\section{References}

Abzug C, Maeda M, Peterson BW, Wilson VJ (1974) Cervical branching of lumbar vestibulospinal axons. J Physiol 243:499-522.

Albus JA (1971) A theory of cerebellar function. Math Biosci 10:25-61.

Anderson RW, Keller EL, Gandhi NJ, Das S (1998) Two-dimensional saccade-related population activity in superior colliculus in monkey. J Neurophysiol 80:798-817.

Barash S, Melikyan A, Sivakov A, Zhang M, Glickstein M, Thier P (1999) Saccadic dysmetria and adaptation after lesions of the cerebellar cortex. J Neurosci 19:10931-10939.

Carey MR, Medina JF, Lisberger SG (2005) Instructive signals for motor learning from visual cortical area MT. Nat Neurosci 8:813-819.

Frens MA, van Opstal AJ (1994) Transfer of short-term adaptation in human saccadic eye movements. Exp Brain Res 100:293-306.

Fuchs AF, Robinson DA (1966) A method for measuring horizontal and vertical eye movement chronically in the monkey. J Appl Physiol 21:1068-1070.

Goldberg ME, Wurtz RH (1972) Activity of superior colliculus in behaving monkey. I. Visual receptive fields of single neurons. J Neurophysiol 35:542-559.

Harting JK (1977) Descending pathways from the superior collicullus: an autoradiographic analysis in the rhesus monkey (Macaca mulatta). J Comp Neurol 173:583-612.

Helmchen C, Straube A, Büttner U (1994) Saccadic lateropulsion in Wallenberg's syndrome may be caused by a functional lesion of the fastigial nucleus. J Neurol 241:421-426.

Huerta MF, Harting JK (1984) The mammalian superior colliculus studies of its morphology and connections. In: Comparative neurology of the optic tectum (Vanegas H, ed), pp 687-773. New York: Plenum.

Ito M (2000) Mechanisms of motor learning in the cerebellum. Brain Res 886:237-245.

Judge SJ, Richmond BJ, Chu FC (1980) Implantation of magnetic search coils for measurement of eye position: an improved method. Vision Res 20:535-538.

Kaku Y, Yoshida K, Iwamoto Y (2009) Learning signals from the superior colliculus for adaptation of saccadic eye movements in the monkey. J Neurosci 29:5266-5275.

Kojima Y, Yoshida K, Iwamoto Y (2007) Microstimulation of the midbrain tegmentum creates learning signals for saccade adaptation. J Neurosci 27:3759-3767.

Kralj-Hans I, Baizer JS, Swales C, Glickstein M (2007) Independent roles for the dorsal paraflocculus and vermal lobule VII of the cerebellum in visuomotor coordination. Exp Brain Res 177:209-222.

Marr DA (1969) A theory of cerebellar cortex. J Physiol 202:437-470.
Martin TA, Keating JG, Goodkin HP, Bastian AJ, Thach WT (1996) Throwing while looking through prisms. I. Focal olivocerebellar lesions impair adaptation. Brain 119:1183-1198.

Mays LE, Sparks DL (1980) Dissociation of visual and saccade-related responses in superior colliculus neurons. J Neurophysiol 43:207-232.

McLaughlin SC (1967) Parametric adjustment in saccadic eye movements. Percep Psychophys 2:359-362.

Mustari MJ, Fuchs AF, Kaneko CR, Robinson FR (1994) Anatomical connections of the primate pretectal nucleus of the optic tract. J Comp Neurol 349:111-128.

Noto CT, Watanabe S, Fuchs AF (1999) Characteristics of simian adaptation fields produced by behavioral changes in saccade size and direction. J Neurophysiol 81:2798-2813.

Optican LM, Robinson DA (1980) Cerebellar-dependent adaptive control of primate saccadic system. J Neurophysiol 44:1058-1076.

Robinson DA (1972) Eye movements evoked by collicular stimulation in the alert monkey. Vision Res 12:1795-1808.

Robinson FR, Noto CT, Bevans SE (2003) Effect of visual error size on saccade adaptation in monkey. J Neurophysiol 90:1235-1244.

Shafer JL, Noto CT, Fuchs AF (2000) Temporal characteristics of error signals driving saccadic gain adaptation in the macaque monkey. J Neurophysiol 84:88-95.

Soetedjo R, Fuchs AF (2006) Complex spike activity of Purkinje cells in the oculomotor vermis during behavioral adaptation of monkey saccades. J Neurosci 26:7741-7755.

Soetedjo R, Kojima Y, Fuchs AF (2008) Complex spike activity in the oculomotor vermis of the cerebellum: a vectorial error signal for saccade motor learning? J Neurophysiol 100:1949-1966.

Sparks DL, Mays LE (1980) Movement fields of saccade-related burst neurons in the monkey superior colliculus. Brain Res 190:39-50.

Straube A, Fuchs AF, Usher S, Robinson FR (1997) Characteristics of saccadic gain adaptation in rhesus macaques. J Neurophysiol 77:874-895.

Straube A, Deubel H, Ditterich J, Eggert T (2001) Cerebellar lesions impair rapid saccade amplitude adaptation. Neurology 57:2105-2108.

Takagi M, Zee DS, Tamargo RJ (1998) Effects of lesions of the oculomotor vermis on eye movements in primate: saccades. J Neurophysiol 80:19111931.

Takeichi N, Kaneko CR, Fuchs AF (2007) Activity changes in monkey superior colliculus during saccade adaptation. J Neurophysiol 97:4096-4107.

Waespe W, Baumgartner R (1992) Enduring dysmetria and impaired gain adaptivity of saccadic eye movements in Wallenberg's lateral medullary syndrome. Brain 115:1123-1146. 